\title{
US politics and ideology enter exam rooms
}

$\mathrm{S}$ eeing a doctor for a legal abortion in some parts of the United States can be a bit like getting arrested. Never mind the right to remain silent. You may be told you have the right to close your eyes and cover your ears for the ultrasound presentation required by law.

In such cases, it's not just the doctor and the patient in the room. In effect, it's the state government, too.

More than 40 years after the US Supreme Court legalized abortion, Republican governors and legislatures are pressing the antiabortion cause with logistical hurdles and edicts in the examination room.

A growing number of states have set controls on abortion counselling and care that are not seen in other areas of medicine. This has aggravated concerns that a political agenda is not just driving health care policy, but dictating what happens between patient and provider at the point of delivery.

A new report by the National Partnership for Women \& Families, an advocacy group that supports abortion rights, says a majority of states, 33, have laws controlling what providers can say or do with patients seeking a legal abortion.

"State legislators all across the country are enacting laws that mandate how doctors, nurses and other health care providers have to practise medicine, without regard to the providers' professional judgment," says Judith Lichtman, the partnership's senior adviser and former president. "Once one interposes politics in the patient-provider relationship, it's a rather slippery slope."

Although rooted in the abortion debate, such laws are not limited to it. In Florida, doctors must watch what they say to patients about gun safety, lest they run afoul of a law nicknamed "Docs vs. Glocks."

The law, upheld by a federal court July 25 , prohibits doctors from routinely asking patients if there are guns at home and from "harassing" them about it. That puts doctors in a tight spot because the American Academy of Pediatrics calls on pediatricians to counsel patients and their parents about safe use and storage of firearms.

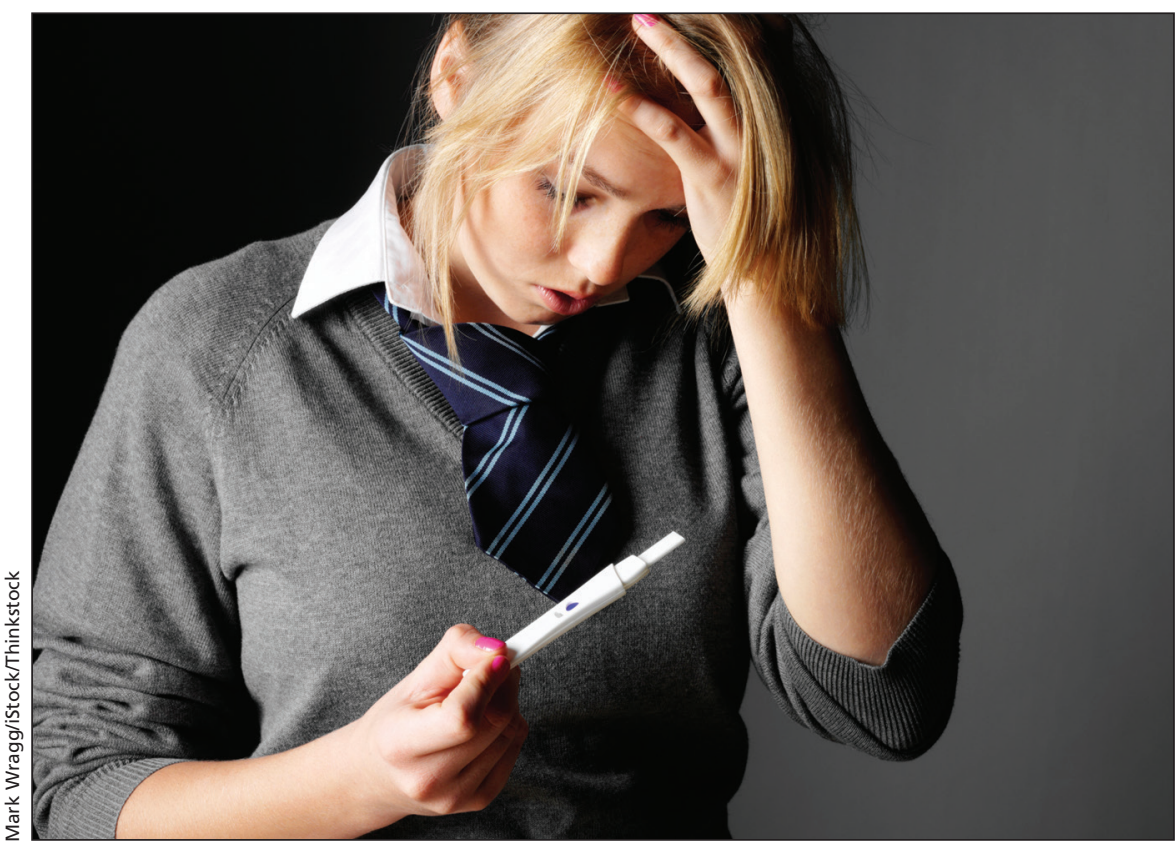

Some state governments are pressing the antiabortion cause with logistical hurdles and edicts in the medical exam room.

The trend has raised red flags at the American Medical Association and the American College of Obstetricians and Gynecologists. They jointly opposed a particularly restrictive Texas abortion law, contending that, "legislatures should not interfere with patient care, medical decisions, and the patient-physician relationship."

In Missouri, women seeking an abortion must first receive in-person counselling directed by the state, then wait 24 hours. They must also have an ultrasound and be offered a chance to hear the fetal heartbeat.

Dr. Colleen McNicholas of St. Louis, who works in Missouri's only freestanding abortion clinic, says she is forced to give patients state-drafted information that overstates the risk of abortion and contains 14 lines the patient must initial. One line verifies that she knows her baby can get state support if she decides against abortion.

"The group of people who get most upset about the restrictions are people who never really thought about the issue of abortion until they find themselves needing one," she says. "They get overwhelmed with all the hoops."

Five states go beyond Missouri and require an ultrasound to be shown to the patient, not just offered. Defending such legislation in Pennsylvania in 2012, Gov. Tom Corbett took heat when he said: "I don't know how you can make anybody watch. Because you just have to close your eyes."

The National Partnership's report also documented that 30 states require waiting periods, and 11 insist on in-person counselling, meaning at least two clinic visits. As well, 13 states require an ultrasound before an abortion. Providers in 28 states must give patients state-written information, some of it falsely linking abortion to breast cancer and fertility problems, doctors say. Five states narrow the use of drug-induced abortion by requiring adherence to an old protocol that physicians consider outdated and needlessly restrictive.

Leaders in some states are open about their ultimate goal. Enacting restrictions in April, Mississippi Gov. Phil Bryant declared: "We're going to try to end abortion in Mississippi."

To Lichtman, the encroachments beg two questions: "How do politicians get into the exam room in the first place? And how fast can we get them out?" Cal Woodward, Washington DC

CMAJ 2014. DOI:10.1503/cmaj.109-4866 